\title{
Efecto del tabaquismo, los síntomas respiratorios y el asma sobre la espirometría de adultos de la Ciudad de México
}

\author{
Justino Regalado-Pineda, M en C,(1) Alejandro Gómez-Gómez, MC, (1) Javier Ramírez-Acosta, MC,(2) \\ Juan Carlos Vázquez-García, M en C. ${ }^{(1,2)}$
}

\section{Regalado-Pineda J, Gómez-Gómez A, Ramírez-Acosta J,Vázquez-García JC. Efecto del tabaquismo, los síntomas respiratorios y el asma sobre la espirometría de adultos de la Ciudad de México. Salud Publica Mex 2005;47:327-334.}

\section{Resumen}

Objetivo. Evaluar el efecto del tabaquismo, los síntomas respiratorios y el asma sobre la función pulmonar espirométrica en población adulta mexicana. Material y métodos. Se generaron ecuaciones de predicción basadas en modelos de regresión lineal múltiple para la capacidad vital forzada (FVC), el vollumen espiratorio forzado al primer segundo $\left(F E V_{1}\right)$ y $F E V_{1} / F V C$ de espirometrías obtenidas de adultos que acuden a evaluación de salud y se determinó el efecto del tabaquismo, los síntomas respiratorios y el asma sobre los modelos de estos parámetros. Resultados. Se estudiaron 919 sujetos de entre 14 y 86 años de edad. El asma disminuye la FVC y el FEV, en hombres con un cambio en la $R^{2}<1 \%$. Los síntomas respiratorios reducen $\mathrm{FEV}_{1} / \mathrm{FVC}$ en ambos sexos. El tabaquismo disminuye significativamente el FEV en las mujeres. Conclusiones. El antecedente de asma disminuyó discretamente la función pulmonar en hombres, mientras que la historia de tabaquismo redujo el $\mathrm{FEV}_{1}$, particularmente en las mujeres de la población que se estudió.

Palabras clave: espirometría; valores de referencia; asma; tabaquismo
Regalado-Pineda J, Gómez-Gómez A,

Ramírez-Acosta J,Vázquez-García JC.

Effect of tobacco smoking, respiratory symptoms

and asthma on spirometry among adults attending

a check-up clinic in Mexico City.

Salud Publica Mex 2005;47:327-334.

\begin{abstract}
Objective. To evaluate the effect of tobacco smoking, respiratory symptoms, and asthma on lung function among Mexican adults who were evaluated during a medical exam in a private health clinic. Material and Methods. Reference prediction equations were generated for spirometry parameters [forced vital capacity (FVC), forced expired volume in one second $\left(\mathrm{FEV}_{\mathrm{f}}\right)$ and $\mathrm{FEV} / \mathrm{FVC}$ ] based on multiple linear regression models. The effect of tobacco smoking, respiratory symptoms and asthma on these equations were explored. Results. Spirometry tests were performed on 919 subjects from 14 to 86 years of age. Asthma decreased FVC and $\mathrm{FEV}$, in men with a $R^{2}$ change $<1 \%$. Respiratory symptoms decreased the FEV $/$ FVC ratio in both sexes. Tobacco smoking was associated with a significant reduction in FEV in women. Conclusions. Asthma lightly reduced lung function in males while tobacco smoking decreased $\mathrm{FEV}_{\text {, }}$, particularly in females.
\end{abstract}

Keywords: spirometry; reference values; asthma; smoking

(I) Departamentos de Fisiología Respiratoria y de Investigación en Tabaquismo, Instituto Nacional de Enfermedades Respiratorias, México, D.F., México.

(2) Departamento de Apoyo Respiratorio, Hospital Angeles del Pedregal, México, D.F., México.

Fecha de recibido: 10 de noviembre de 2004 - Fecha de aprobado: 17 de agosto de 2005 Solicitud de sobretiros: M. en C. Juan Carlos Vázquez García, Calzada de Tlalpan 4502. Sección XVI, I 4080 México, D.F. Correo electrónico: jcvaz@mexis.com 
L a espirometría en la actualidad se considera la mejor prueba de función mecánica respiratoria por ser la más simple, accesible y reproducible. ${ }^{1-3}$ Además, los resultados de la prueba se correlacionan bien con la morbilidad y la expectativa de vida.

Los principales determinantes de la función pulmonar son el género, la talla, la edad, el origen étnico y los factores técnicos. En conjunto, las variables mencionadas explican cerca de $70 \%$ de la variabilidad total, en tanto que no se explica el restante $30 \%$ de la variabilidad de la función pulmonar entre sujetos. ${ }^{4-7}$ Actualmente existe suficiente evidencia en la literatura respecto al efecto racial o étnico de la función pulmonar en diferentes grupos de población como los sujetos de raza negra, ${ }^{8}$ habitantes de las islas del Pacífico, ${ }^{9}$ nativos de Norteamérica $^{10}$ y Nueva Zelanda, ${ }^{11}$ orientales $^{12,13}$ y latinos en Estados Unidos de América (EUA).14,15

Los valores normales para espirometría, también llamados de referencia o predichos, que se usan en la actualidad en los laboratorios de función pulmonar en México, provienen de poblaciones externas, generalmente de Norteamérica y Europa. ${ }^{16-18}$ Se trata de poblaciones de origen caucásico cuyos patrones de función pulmonar pueden ser diferentes de los observados en la población mestiza mexicana.

En un estudio previo ${ }^{19}$ se observó que los valores estimados de la capacidad vital forzada (FVC) y el volumen espiratorio forzado al primer segundo $\left(\mathrm{FEV}_{1}\right)$ por medio de una ecuación derivada de sujetos mexicanos se encuentran por arriba de los obtenidos en otras poblaciones como las estudiadas por Quanjer ${ }^{18}$ y Coultas, ${ }^{14,15}$ lo que significa que estas ecuaciones externas subestiman los valores espirométricos ideales. En contraste, las ecuaciones desarrolladas por Hankinson ${ }^{20}$ en población de origen mexicano de EUA y $\mathrm{Crapo}^{16}$ en población caucásica se asemejan más a la función espirométrica de una población de trabajadores mexicanos. ${ }^{19}$ Sin embargo, en esta población no se discriminó a los fumadores ni a los sujetos con síntomas respiratorios o diagnóstico de asma. En el presente estudio evaluamos el impacto de los síntomas respiratorios, el asma diagnosticada y los antecedentes de tabaquismo sobre la función pulmonar en un grupo de sujetos que acudieron a valoración de salud en un hospital privado de la Ciudad de México. Además, se describen ecuaciones de referencia específica generadas en un subgrupo de sujetos aparentemente sanos, sin síntomas respiratorios y nunca fumadores.

\section{Pacientes y métodos}

Se revisaron de manera retrospectiva todas las espirometrías realizadas a personas que acudieron a una va- loración médica de rutina en un hospital privado del sur de la Ciudad de México durante los meses de marzo a diciembre de 1999. Se consideró a todos los sujetos que solicitaban una espirometría, incluso a personas con historia de tabaquismo, síntomas respiratorios o historia de asma previamente diagnosticada por un médico. Se excluyeron a todos los sujetos con otras enfermedades crónicas concomitantes o que no contaban con una espirometría adecuada de acuerdo con los lineamientos sugeridos por la Sociedad Americana del Tórax (ATS, por sus siglas en inglés). ${ }^{3}$

Todos los participantes completaron una espirometría, así como un cuestionario estandarizado ${ }^{21,22}$ que explora en forma dicotómica la historia de tabaquismo, síntomas respiratorios (tos, expectoración, sibilancias o disnea), enfermedades respiratorias agudas (infección respiratoria de cualquier tipo presente dentro de las cuatro semanas previas al estudio) y enfermedades crónicas limitantes. La presencia de síntomas respiratorios se consideró como positiva cuando el individuo respondía afirmativamente a cualquiera de las preguntas referentes a síntomas.

La espirometría se realizó en el laboratorio de función pulmonar con una temperatura ambiente controlada, usando un espirómetro computarizado con medición de flujos por medio de neumotacógrafo (Medical Graphics Corporation System 1070 Series 2E/ 1085, St. Paul MN, USA). Este equipo cumple con los criterios de desempeño sugeridos por la ATS. ${ }^{3}$ El procedimiento siguió los lineamientos recomendados, y el personal técnico que realizó la prueba se capacitó previamente, de acuerdo con los lineamientos del Instituto Norteamericano de Salud y Seguridad Ocupacional (NIOSH, por sus siglas en inglés).

El equipo fue calibrado diariamente con una jeringa estándar de 31 y la variabilidad máxima permitida fue de 3\% (90 ml). Los valores se ajustaron a condiciones estándar de presión barométrica y temperatura corporal (BTPS). La presión barométrica promedio fue de $583 \mathrm{mmHg}$ para la altitud de la Ciudad de México $(2240 \mathrm{~m})$ y la temperatura ambiente osciló entre $20-24{ }^{\circ} \mathrm{C}$ durante el periodo de estudio.

Todos los participantes realizaron las pruebas sentados o parados y utilizando pinzas nasales. Se obtuvieron al menos tres maniobras espiratorias forzadas aceptables y reproducibles de acuerdo con lo sugerido por la ATS. La calidad de la espirometría se revisó después de cada maniobra espiratoria, y el técnico descartaba los esfuerzos que no cumplieron los criterios de aceptabilidad. Además, el equipo señala automáticamente cuando el esfuerzo espiratorio no es satisfactorio o cuando la variabilidad entre las dos mejores pruebas excede el máximo permitido. Como estrate- 
gia adicional de control de calidad se aplicaron los criterios de Enright ${ }^{23,24}$ basados en la reproducibilidad intraprueba. De esta manera se revisa nuevamente la reproducibilidad entre los tres esfuerzos espiratorios y se selecciona a los dos mejores. Se conservaron solamente las espirometrías con calificación A o B, que implican una gran similitud entre las dos mejores maniobras espiratorias y se consideran más estrictas que los lineamientos sugeridos por la ATS [A = variabilidad del flujo espiratorio máximo (PEFR) $<5 \%$, variabilidad $\mathrm{FEV}_{1}<5 \%$ o $100 \mathrm{ml}$, variabilidad de $\mathrm{FVC}<3 \%$; $\mathrm{B}=$ variabilidad PEFR $>5 \%<10 \%$, variabilidad del $\mathrm{FEV}_{1}<5 \%$ o $100 \mathrm{ml}$, variabilidad de $\mathrm{FVC}>3 \%<5 \%$ ].

\section{Análisis estadístico}

Las principales variables se describen como promedios y desviación estándar o como medianas e intervalos cuando la distribución no fue normal. Para la comparación entre grupo se utilizó la prueba t para muestras independientes o su equivalente no paramétrico.

Se generaron valores de referencia separadas por género para $\mathrm{FVC}, \mathrm{FEV}_{1}$ y $\mathrm{FEV}_{1} / \mathrm{FVC}$ mediante modelos de regresión lineal múltiple (MRLM). Para cada modelo se tomaron como covariables la estatura, la edad, la presencia de síntomas respiratorios, el diagnóstico médico de asma y el índice de masa corporal (IMC). Para estimar la contribución de cada covariable a la varianza del modelo se siguió el método de MRLM por pasos. La relación entre $\mathrm{FVC}, \mathrm{FEV}_{1}$ y $\mathrm{FEV}_{1} / \mathrm{FVC}$ con las variables independientes se exploró de manera lineal y no lineal de acuerdo con modelos de potencias de edad y talla. ${ }^{25}$ Se considera más apropiada la relación no lineal cuando el modelo incrementa el coeficiente de determinación $\left(R^{2}\right)$ en conjunto con una disminución significativa de la suma de cuadrados de los residuales (MSR). Posteriormente, se calcularon valores predichos para cada parámetro espirométrico, así como el porcentaje del predicho para cada sujeto de acuerdo con la ecuación interna y cada una de las ecuaciones externas comparadas. Para cada grupo de ecuaciones se calculó el valor de los residuales y se analizaron las diferencias entre las ecuaciones externas y la ecuación interna mediante el coeficiente de correlación intraclase (CCI) ${ }^{25}$ Se consideró como estadísticamente significativo un valor de $p<0.05$. Para el análisis estadístico se utilizó el programa SPSS para Windows versión 9.0.

\section{Resultados}

Durante el periodo de estudio se refirieron un total de 1401 personas entre 14 y 86 años de edad. Se excluyeron 67 sujetos $(4.7 \%)$ por datos insuficientes en el cuestionario y 415 sujetos (30\%) que excedieron la va- riabilidad intraprueba máxima permitida (con calificación $C$ de Enright), por lo que en el análisis final se incluyeron 919 sujetos (675 hombres, 244 mujeres). La prevalencia de tabaquismo, síntomas respiratorios y el diagnóstico médico previo de asma fue similar de acuerdo con los grados de calidad de las espirometrías (clases A, B o C de Enright). En función de estos mismos, la prevalencia de tabaquismo fue de 42 , 48 y $41 \%$; la de síntomas respiratorios (tos aguda o crónica, expectoración, sibilancias o disnea), de 4,5 y $6 \%$, y la de asma; de 9,9 y $8 \%$, respectivamente. El cuadro I muestra las características generales de los sujetos incluidos según el género. Ambos grupos fueron similares en edad; como era de esperarse, los hombres mostraron valores promedio mayores de estatura, peso y función pulmonar. No se observaron diferencias significativas para síntomas respiratorios o historia de asma entre ambos géneros. El tabaquismo fue más frecuente entre los hombres en comparación con las mujeres ( $48 \%$ vs. $35 \%, p=0.001)$. Sin embargo, la intensidad del tabaquismo, medido como índice paquetes-año, fue similar entre hombres y mujeres $(p>0.1)$

Para la generación de las ecuaciones de referencia se utilizaron sólo 490 sujetos (340 hombres y 150 mujeres) sin historia de síntomas respiratorios, asma o tabaquismo. El análisis de residuales mostró una distribución razonablemente homogénea para $\mathrm{FVC} /$ talla

\section{Cuadro I \\ Características generales de los pacientes de un hospital privado de la Ciudad de México A LOS QUE SE LES HIZO ESPIROMETRÍA}

\begin{tabular}{lcc} 
& $\begin{array}{c}\text { Hombres } \\
n=675\end{array}$ & $\begin{array}{c}\text { Mujeres } \\
n=244\end{array}$ \\
Edad $($ años $)$ & $43 \pm 10$ & $43 \pm 12$ \\
\hline Talla $(\mathrm{cm})$ & $172 \pm 7$ & $161 \pm 7^{*}$ \\
\hline Peso $(\mathrm{kg})$ & $77 \pm 12$ & $62 \pm 12^{*}$ \\
\hline $\mathrm{IMC}\left(\mathrm{kg} / \mathrm{m}^{2}\right)$ & $26 \pm 4$ & $24 \pm 4^{*}$ \\
\hline $\mathrm{FVC}(\mathrm{l})$ & $4.62 \pm 0.80$ & $3.42 \pm 0.60^{*}$ \\
\hline $\mathrm{FEV},(\mathrm{l})$ & $3.81 \pm 0.70$ & $2.86 \pm 0.60^{*}$ \\
\hline $\mathrm{FEV}, \mathrm{FVC}, \%$ & $82.5 \pm 5.4$ & $83.6 \pm 5.5$ \\
\hline Síntomas respiratorios & $26(4 \%)$ & $16(7 \%)$ \\
\hline Historia de asma & $6 \mathrm{I}(9 \%)$ & $20(8 \%)$ \\
\hline Historia de tabaquismo & $322(48 \%)$ & $85(35 \%)^{\ddagger}$ \\
\hline Intensidad de tabaquismo, paquetes/año & $5(\mathrm{I}-120)$ & $6(\mathrm{I}-120)$
\end{tabular}

Nota: Los valores se presentan como proporciones o promedios y desviaciones estándar, excepto para intensidad de tabaquismo, que se presenta como mediana y valores mínimo-máximo.

$* p<0.00$ I por prueba $t$ de Student

$\ddagger p=0.00$ I por prueba de ji cuadrada 
y $\mathrm{FEV}_{1}$ / talla, con lo que se cumple el supuesto de homocedasticidad. Asimismo, se analizaron relaciones no lineales entre cada parámetro ajustando por edad y talla. Debido a que no se modificaron los coeficientes de regresión se decidió emplear modelos lineales. En el cuadro II se muestran los coeficientes de regresión lineal para $\mathrm{FVC}, \mathrm{FEV}_{1}$ y $\mathrm{FEV}_{1} / \mathrm{FVC}$ y la figura 1 ilustra la relación entre $\mathrm{FVC}$ y $\mathrm{FEV}_{1}$ con la talla y la edad de acuerdo con la ecuación interna y diferentes ecuaciones externas de uso común. Las ecuaciones con mejor

\section{Cuadro II \\ EFECTO DE SíNTOMAS RESPIRATORIOS, ASMA Y TABAQUISMO SOBRE FVC, FEV, Y LA RELACIÓN FEV IFVC. PACIENTES DE UN HOSPITAL PRIVADO de la Ciudad de México}

Variable Coeficiente $\mathrm{R}^{2} \quad \mathrm{EE}$

Hombres $(N=675)$

FVC

\begin{tabular}{|c|c|c|c|c|}
\hline Talla (cm) & 0.07 & 0.48 & 0.60 & $<0.001$ \\
\hline Edad (años) & -0.02 & 0.55 & 0.56 & $<0.001$ \\
\hline IMC $\left(\mathrm{kg} / \mathrm{m}^{2}\right)$ & -0.19 & 0.56 & 0.56 & 0.012 \\
\hline Asma & -0.20 & 0.57 & 0.55 & 0.015 \\
\hline \multicolumn{5}{|l|}{$\mathrm{FEV}_{1}$} \\
\hline Talla $(\mathrm{cm})$ & 0.05 & 0.41 & 0.53 & $<0.001$ \\
\hline Edad (años) & -0.02 & 0.53 & 0.48 & $<0.001$ \\
\hline Asma & -0.18 & 0.54 & 0.47 & 0.013 \\
\hline \multicolumn{5}{|l|}{$\mathrm{FEV}_{1} / \mathrm{FVC}$} \\
\hline Edad (años) & -0.14 & 0.04 & 5.33 & $<0.001$ \\
\hline Talla (cm) & -0.13 & 0.07 & 5.26 & $<0.001$ \\
\hline Síntomas & -4.31 & 0.09 & 5.20 & $<0.001$ \\
\hline
\end{tabular}

Mujeres $(N=244)$

FVC

\begin{tabular}{lllll} 
Talla $(\mathrm{cm})$ & 0.06 & 0.38 & 0.60 & $<0.001$ \\
\hline Edad (años) & -0.03 & 0.57 & 0.51 & $<0.001$
\end{tabular}

FEV,

\begin{tabular}{ccccc} 
Talla $(\mathrm{cm})$ & 0.05 & 0.35 & 0.55 & $<0.001$ \\
\hline Edad (años) & -0.03 & 0.55 & 0.46 & $<0.001$ \\
\hline $\begin{array}{c}\text { Paquetes/año } \\
\text { FEV } / \text { FVC }\end{array}$ & 0.01 & 0.58 & 0.45 & 0.036 \\
Edad (años) & -0.09 & 0.03 & 5.38 & 0.001 \\
\hline Síntomas & -2.86 & 0.05 & 5.34 & 0.028
\end{tabular}

Notas: Los resultados se presentan por separado para hombres y mujeres y se basan en modelos de regresión lineal múltiple. Como covariables se incluyeron en el modelo la edad, la talla, los síntomas respiratorios, el diagnóstico de asma, el IMC y el tabaquismo.

EE: error estándar de la estimación

IMC: índice de masa corporal

FVC: capacidad vital forzada

$\mathrm{FEV}_{1}$ : volumen respiratorio forzado al primer segundo. desempeño fueron la de Hankinson, ${ }^{20} \mathrm{Crapo}^{16}$ y la de Pérez-Padilla. ${ }^{19}$ Los coeficientes de correlación interclase (CCI) para residuales entre la ecuación interna y la ecuación de Pérez-Padilla fueron mayores a 0.97 en el grupo de mujeres, mientras que en los hombres la aplicación de esta ecuación tiende a ser inadecuada, especialmente en la estimación de la FVC, $\mathrm{CCI}=0.85$. Otras ecuaciones de uso común mostraron un desempeño bajo con base en los CCI. Llama la atención la escasa correlación entre los valores observados en la población estudiada y los estimados por Coultas ${ }^{14,15}$ descritos en población de origen latinoamericano en Nuevo México, EUA; CCI de 0.83 y 0.77 para $\mathrm{FEV}_{1}$ en función de la edad en hombres y mujeres, respectivamente.

En el cuadro III se presenta el efecto de los síntomas respiratorios, asma, tabaquismo y obesidad sobre FVC, $\mathrm{FEV}_{1}$ y la relación $\mathrm{FEV}_{1} / \mathrm{FVC}$. La FVC en los hombres se encuentra determinada principalmente por la edad y la talla, $R^{2}=0.55$. La obesidad medida como el IMC, presentó un efecto negativo sobre la FVC en el grupo de hombres. El asma presentó igualmente un efecto negativo sobre la FVC en hombres; sin embargo, el cambio de la $R^{2}$ fue menor a $1 \%$. En el caso de las mujeres, la FVC se encuentra determinada principalmente por la estatura y la edad, sin que se modifique por la presencia de síntomas respiratorios, asma, obesidad o tabaquismo.

El $\mathrm{FEV}_{1}$ en los hombres se encuentra determinado principalmente por la estatura y la edad, $R^{2}=0.53$. El

\section{Cuadro III \\ Coeficientes de Regresión LiNeAL múltiple PARA LAS VARIABLES ESPIROMÉTRICAS}

$$
\text { Constante Edad Talla } \mathrm{R}^{2} \quad \mathrm{EE}
$$

Hombres $(N=340)$

\begin{tabular}{llllll}
$\mathrm{FVC}(\mathrm{I})$ & -5.26 & -0.03 & 0.06 & 0.52 & 0.52 \\
\hline $\mathrm{FEV}_{1}(\mathrm{I})$ & $-3.2 \mathrm{I}$ & -0.03 & 0.05 & $0.5 \mathrm{I}$ & 0.44 \\
\hline $\mathrm{FEV}_{\mathrm{I}} / \mathrm{FVC}(\%)$ & 88.02 & -0.12 & - & 0.04 & 5.38
\end{tabular}

Mujeres $(N=150)$

\begin{tabular}{llllll} 
FVC (I) & -1.09 & -0.02 & 0.04 & 0.59 & 0.34 \\
\hline FEV $_{1}(\mathrm{I})$ & -0.36 & -0.02 & 0.03 & 0.60 & 0.30 \\
\hline FEV $_{1}$ IFVC (\%) & 99.14 & -0.07 & - & 0.04 & 4.42
\end{tabular}

Notas: Los resultados se presentan por separado para 340 hombres y I 50 mujeres sin síntomas respiratorios ni historia de asma o tabaquismo. Se basan en modelos de regresión lineal múltiple; como covariables se incluyeron en el modelo la edad y la talla. Sólo se incluyen los coeficientes de covariables estadísticamente significativas $(p<0.05)$

EE: error estándar de la estimación

FVC: capacidad vital forzada

$\mathrm{FEV}_{1}$ : volumen respiratorio forzado al primer segundo 

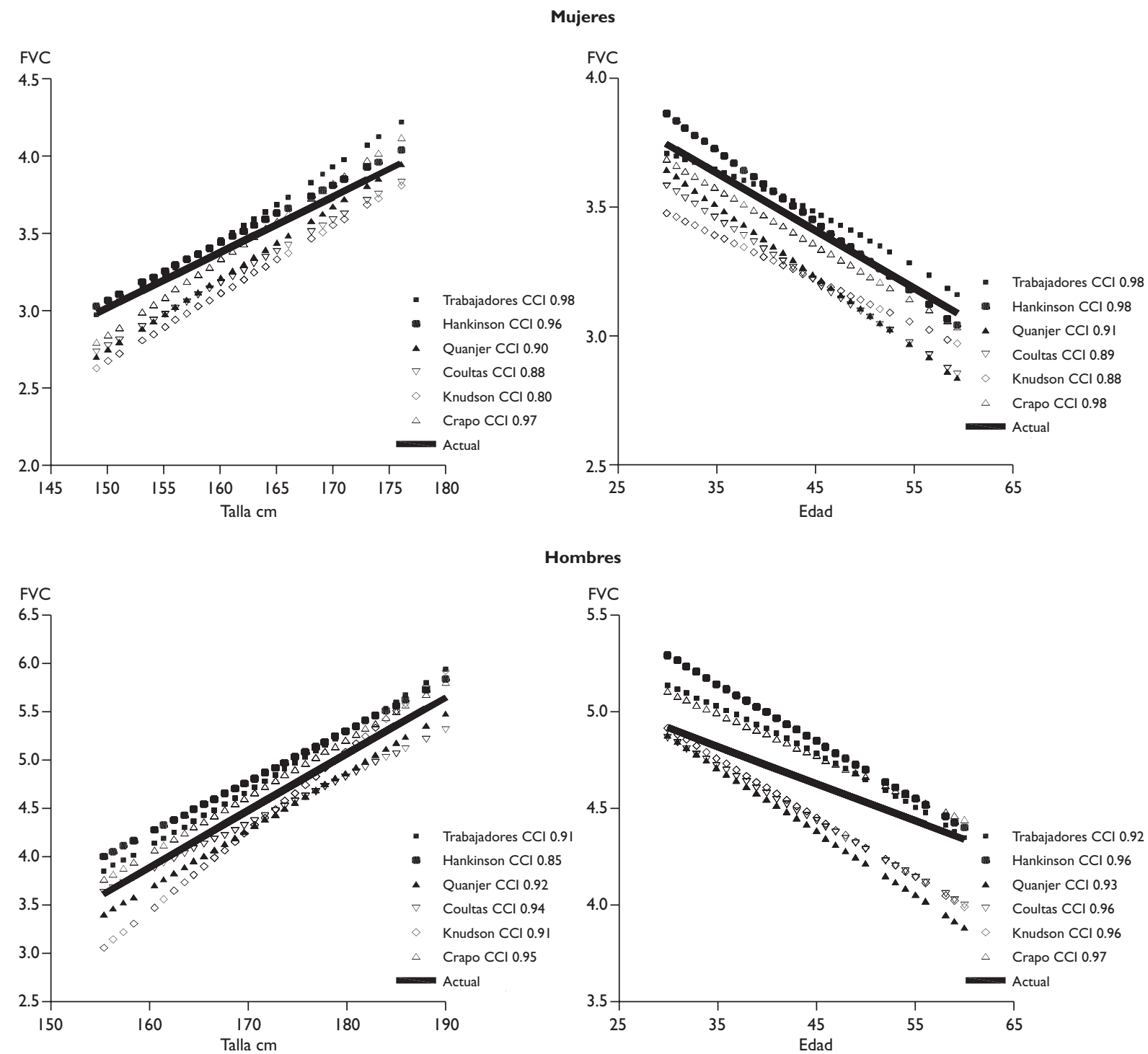

Figura I. La figura muestra la estimación de FVC en hombres (PANel superior) y en mujeres (PANel iNferior)

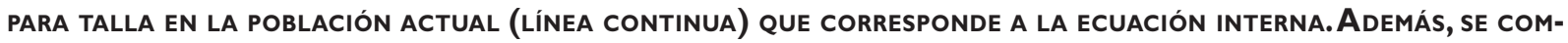
para con seis eCuaciones de Referencia externa. Se puede observar como las ecuaciones de HaNkinson, trabajadores mexicanos (Pérez-Padilla y col.) y Crapo son las Que más se asemejan, mientras Que las de KNUdson, COULTAS Y QUANJER TIENDEN A SUBESTIMAR DE MANERA SIGNIFICATIVA LA FUNCIÓN PULMONAR DE LA POBLACIÓN

asma mostró un efecto negativo sobre el $\mathrm{FEV}_{1}$ con un cambio de la $R^{2}<1 \%$. En las mujeres, el tabaco mostró un efecto negativo importante en la función pulmonar, disminuyendo $6.4 \mathrm{ml}$ por cada paquete-año de incremento en el índice de tabaquismo, y determinó un $2.4 \%$ de la variabilidad del modelo. El efecto es más marcado en mujeres con índice mayor a 15 paquetesaño, que correspondía a una reducción de hasta 167 $\mathrm{ml}$ en el $\mathrm{FEV}_{1}$ (datos no mostrados).
La relación $\mathrm{FEV}_{1} / \mathrm{FVC}$ disminuyó con la edad, la talla y los síntomas respiratorios, tanto en hombres como en mujeres.

El cuadro IV muestra los valores de $\mathrm{FVC}, \mathrm{FEV}_{1}$ y $\mathrm{FEV}_{1} / \mathrm{FVC}$ expresados como por ciento del predicho, así como el valor del percentil 5 (PC5) como indicador del límite inferior de normalidad. En general, la aplicación de las ecuaciones de Quanjer, Coultas y Knudson resulta en una subestimación de los valores de FVC, 
$\mathrm{FEV}_{1}$ y $\mathrm{FEV}_{1} / \mathrm{FVC}$, lo que, a su vez, produce valores predichos por arriba de $100 \%$. La subestimación de los parámetros espirométricos es más marcada cuando se aplican los valores de referencia de Quanjer. Nuevamente la ecuación de mexicoamericanos de Hankinson y colaboradores es la que mejor se ajusta a la población estudiada, de tal manera que se observan valores muy cercanos a 100\%. La aplicación de las ecuaciones de Quanjer, Coultas y Knudson para estimar FVC y FEV 1 resulta en valores del PC5 cercanos a 90\% del predicho; en cambio, al aplicar la ecuación de Crapo, Pérez-Padilla y Hankinson, el PC5 se ubica alrededor de $80 \%$ del predicho.

\begin{tabular}{|c|c|c|c|}
\hline \multicolumn{4}{|c|}{ Cuadro IV } \\
\hline \multicolumn{4}{|c|}{ LÍMITES INFERIORES DE NORMALIDAD (PERCENTIL 5) } \\
\hline \multirow{3}{*}{\multicolumn{4}{|c|}{$\begin{array}{l}\text { PARA POR CIENTOS DEL PREDICHO DE DIFERENTES } \\
\text { ECUACIONES DE REFERENCIA EN PACIENTES } \\
\text { DE UN HOSPITAL PRIVADO DE LA CD. DE MÉXICO, }\end{array}$}} \\
\hline & & & \\
\hline & & & \\
\hline Ecuación & Variable & Hombres & Mujeres \\
\hline \multirow[t]{3}{*}{ Quanjer } & FVC & 105 [87] & 106 [87] \\
\hline & $\mathrm{FEV}_{1}$ & 109 [89] & III [89] \\
\hline & $\mathrm{FEV}_{\mathrm{I}} / \mathrm{FVC}$ & 105 [94] & 104 [93] \\
\hline \multirow[t]{2}{*}{ Coultas } & FVC & 104 [86] & 107 [89] \\
\hline & $\mathrm{FEV}_{\text {, }}$ & $106[88]$ & $107[86]$ \\
\hline \multirow[t]{3}{*}{ Knudson } & FVC & 104 [85] & 106 [89] \\
\hline & $\mathrm{FEV}_{\text {, }}$ & $105[85]$ & $107[87]$ \\
\hline & $\mathrm{FEV}_{\mathrm{I}} / \mathrm{FVC}$ & 101 [91] & 74 [67] \\
\hline \multirow[t]{3}{*}{ Crapo } & FVC & $97[80]$ & 102 [85] \\
\hline & $\mathrm{FEV}_{1}$ & $99[8 \mathrm{I}]$ & $102[83]$ \\
\hline & $\mathrm{FEV}_{\mathrm{I}} / \mathrm{FVC}$ & 102 [9I] & $101[91]$ \\
\hline \multirow[t]{3}{*}{ Pérez-Padilla } & FVC & 95 [78] & 99 [83] \\
\hline & $\mathrm{FEV}_{1}$ & $98[8 I]$ & $102[82]$ \\
\hline & $\mathrm{FEV}_{1} / \mathrm{FVC}$ & 103 [92] & $102[92]$ \\
\hline \multirow[t]{3}{*}{ Hankinson } & FVC & 97 [79] & 108 [91] \\
\hline & $\mathrm{FEV}_{1}$ & $100[81]$ & $101[82]$ \\
\hline & $\mathrm{FEV}_{1} / \mathrm{FVC}$ & 98 [88] & $102[91]$ \\
\hline \multirow[t]{3}{*}{ Estudio actual } & FVC & 100 [82] & $100[84]$ \\
\hline & $\mathrm{FEV}_{1}$ & 100 [82] & $100[81]$ \\
\hline & $\mathrm{FEV}_{1} / \mathrm{FVC}$ & 100 [88] & 100 [79] \\
\hline
\end{tabular}

Nota: Los valores se presentan como promedios y percentil 5 entre paréntesis.

FVC: capacidad vital forzada

$\mathrm{FEV}_{1}$ : volumen respiratorio forzado al primer segundo

\section{Discusión}

El presente estudio describe ecuaciones de referencia para parámetros de función espirométrica y el impacto, sobre las mismas, del asma, los síntomas respiratorios y la historia de tabaquismo, en una en población mexicana con un intervalo amplio de edad (14 a 86 años). Asimismo, con este estudio se confirma que los valores de referencia de población mexicana suelen subestimarse en algunas ecuaciones externas que se utilizan con frecuencia.

Los parámetros espirométricos en ambos géneros de nuestra población se encontraron determinados principalmente por la estatura y la edad, como ha sido descrito en la mayoría de ecuaciones de predicción., ${ }^{2,714-20}$ La presencia de síntomas respiratorios disminuyó la relación $\mathrm{FEV}_{1} / \mathrm{FVC}$, mientras que el antecedente de asma se asoció con una disminución de la FVC y el $\mathrm{FEV}_{1}$ en hombres. Este efecto fue significativo y cercano a los $200 \mathrm{ml}$; sin embargo, la $R^{2}$ no se modificó sustancialmente. El tabaquismo se asoció con una reducción significativa del $\mathrm{FEV}_{1}$ en el grupo de mujeres. Tomando en cuenta su impacto sobre la función pulmonar, es evidente que se deben excluir a los sujetos con síntomas respiratorios para la generación de valores de referencia espirométrica. Xu $X_{,}{ }^{26}$ Scanlon $^{27}$ y Silverman ${ }^{28}$ señalan en sus trabajos diferencias de género en cuanto al impacto del tabaquismo en la función pulmonar, así como mayor susceptibilidad para desarrollar enfermedad pulmonar obstructiva crónica, especialmente en las mujeres. Sin embargo, esta información ha sido cuestionada por Vollmer y colaboradores, ${ }^{29}$ quienes no han demostrado un efecto adverso diferencial entre hombres y mujeres. En este estudio se observó un efecto importante del tabaquismo sobre el $\mathrm{FEV}_{1}$ de las mujeres. Aunque el tabaquismo es más frecuente entre los hombres ( $48 \%$ vs. 35\%, $p=0.01$ ), la intensidad medida como índice paquetes-año fue similar en ambos sexos: mediana 5 (rango 1 a 120) para hombres y mediana de 6 (rango 1 a 120) para mujeres, $p>0.05$. Es de llamar la atención que las mujeres presenten efectos adversos significativos sobre la función pulmonar en relación con el hábito tabáquico, lo que no se observa en los hombres a pesar de que ambos grupos mostraron cifras similares en relación con la dosis de tabaco acumulada (cuadro I). En México no existen informes que señalen el efecto del tabaquismo en la función pulmonar de acuerdo con el género, por lo que los resultados observados sientan un precedente que habrá que confirmar en el futuro mediante estudios diseñados específicamente para demostrar el efecto del tabaquismo en población mexicana. Una forma más adecuada de evaluar el efecto del tabaco sobre 
la función pulmonar sería a través de un estudio longitudinal; sin embargo, un abordaje transversal puede considerarse apropiado para este fin.

Las ecuaciones externas con mejor desempeño al aplicarse en el grupo estudiado fueron la de mexicoamericanos desarrollada por Hankinson y la de Crapo. La ecuación de Pérez-Padilla, generada en trabajadores mexicanos estudiados en el Instituto Nacional de Enfermedades Respiratorias (INER), describe satisfactoriamente la función pulmonar medida como $\mathrm{FVC}_{\mathrm{FEV}}{ }_{1}{ }^{19}$ Estos valores de referencia se ajustan con mayor precisión en las mujeres; en los hombres se observó una tendencia a subestimar los valores predichos de FVC y $\mathrm{FEV}_{1}$. Parte de las diferencias encontradas pueden explicarse por edad y estatura. En el presente estudio el promedio de edad y estatura en hombres fue de 43 años y $172 \mathrm{~cm}$, mientras que en el estudio de trabajadores fue de 51 años y $163 \mathrm{~cm}$.

Las disimilitudes en el estado nutricional pueden explicar algunas de las diferencias encontradas entre la población estudiada y las poblaciones externas de referencia. Actualmente se sabe que el estado nutricional modifica el desarrollo de la caja torácica y la masa muscular, lo que tiene un impacto sobre la función pulmonar. ${ }^{30}$ En sujetos residentes de regiones donde las condiciones socioeconómicas han mejorado rápidamente, se ha observado un efecto positivo a nivel de la función pulmonar y las variables que la determinan, específicamente la estatura. ${ }^{31}$

Previamente, se ha descrito un efecto negativo del IMC sobre la función pulmonar en población de ascendencia latinoamericana; ${ }^{14}$ en el caso de población mexicana, tanto en el estudio de trabajadores como en el grupo analizado, no se observó ninguna asociación independiente entre el IMC y la FVC o FEV 1 cuando se analizan los sujetos sin tabaquismo, diagnóstico de asma o síntomas respiratorios. En el presente estudio, el promedio del IMC fue de 24 en las mujeres y 26 en los hombres. En contraste, la población de trabajadores mostró un promedio de IMC de 28 , tanto en mujeres como hombres. Como se ve, la población de trabajadores tiende a presentar rasgos de sobrepeso; sin embargo, el IMC no modifica por sí solo la función pulmonar en población mexicana. Asimismo, la comparación entre poblaciones puede estar confundida por diferencias en el nivel socioeconómico. Dadas las características de referencia del grupo estudiado, no se realizó ningún tipo de ajuste por nivel socioeconómico, pero el grupo de trabajadores estudiados en el INER en su mayoría son obreros, por lo que se puede esperar que su nivel de ingresos sea menor. Sin embargo, ambas poblaciones coinciden razonablemente en la función pulmonar.
Las ecuaciones externas desarrolladas por Hankinson y Crapo podrían aplicarse a población mexicana similar a la incluida en el presente estudio para estimar valores de referencia espirométrica. Aun cuando ambas ecuaciones de referencia describen aceptablemente la función pulmonar, persisten algunas diferencias. Por otra parte, existe estudios que señalan que la función espirométrica en mujeres mexicanas del área rural expuestas al humo de leña es menor que la estimada por las ecuaciones externas ${ }^{33}$ o que cuestionan la aplicación de ecuaciones externas en población mexicana. ${ }^{34}$ Lo anterior apoya la noción de que la población estudiada presenta características de función pulmonar diferentes de las estudiadas en otras partes y se apoya el concepto de utilizar ecuaciones propias. En suma, se corrobora el pobre desempeño de la mayoría de las ecuaciones externas comúnmente utilizadas para predecir función pulmonar en espirometría cuando se aplican a población mexicana. En general, los valores estimados por las diferentes ecuaciones externas son menores en comparación con los valores estimados con ecuaciones propias, y el efecto de esta subestimación es más evidente a partir de los 40 años. En consecuencia, es importante tomar en cuenta las tendencias señaladas cuando se aplican ecuaciones externas en población mexicana. El cuadro IV resume dichas tendencias y presenta el valor del PC5 como indicador del límite inferior de normalidad. Esto pretende ser una guía práctica para los clínicos que cuentan con equipo al cual se le han adaptado de fábrica diferentes ecuaciones de referencia, para establecer el punto de corte al considerar una prueba como normal o anormal cuando se emplea alguna de las ecuaciones enumeradas en el cuadro.

En conclusión, el presente estudio muestra el impacto negativo de los síntomas respiratorios y el asma sobre la función pulmonar, así como el efecto negativo del tabaquismo en el $\mathrm{FEV}_{1}$ del grupo de mujeres. Las ecuaciones de Hankinson y Crapo pueden aplicarse a población mexicana similar a la estudiada. En caso de contar con equipos que utilizan cualquier otra de las ecuaciones externas analizadas, el valor estimado como por ciento del predicho del PC5 debe ajustarse como punto de corte de normalidad.

\section{Agradecimientos}

Los autores desean manifestar su agradecimiento a los técnicos respiratorios Rogelio Amador Camacho, Jazmín López Luna, Julieta Martínez Pérez, Leticia Núñez López y Javier Sánchez Cuica, del Departamento de Apoyo Respiratorio del Hospital Ángeles del Pedregal, por su valiosa ayuda en la realización de las espirometrías. 


\section{Referencias}

I. Crapo R. Pulmonary function testing. N Engl J Med 1994;33I:25-30.

2. American Thoracic Society. Lung function testing: Selection of reference values and interpretative strategies. Am Rev Respir Dis 1991;144:1202-1218.

3. American Thoracic Society. Standardization of spirometry. 1994 update. Am J Respir Crit Care Med 1995; I52: I 107-I I 36.

4. Becklake M,White N. Sources of variation in spirometric measurements. Identifying the signal and dealing with noise. Occup Med 1993;8:241-264.

5. Künzli U,Ackermann-Liebrich U, Keller R, Perruchoud AP, Schlinder C, SAPALDIA team. Variability of FVC and FEV, due to technician, team, device and subject in an eight-centre study:Three quality control studies in SAPALDIA. Eur Respir J 1995;8:37I-376.

6. Becklake M. Concepts of normality applied to the measurement of lung function. Am J Med 1986;80: I I58-I I64.

7. Clausen J. Prediction of normal values in pulmonary function testing. Clin Chest Med 1989;10:135-143.

8. Enright PL,Arnold A, Manolio TA, Kuller LH. Spirometric reference values for healthy elderly blacks. Chest 1996; I 0:1416-1424.

9. Brown P, Sadowsky D, Gajdusek DC. Ventilatory lung function studies in Pacific Island Micronesians. Am J Epidemiol 1978;108:259-265.

10. Crapo RO, Lockey J, Jensen RL, Elliot CG. Normal spirometric values in healthy American Indians. J Occup Med 1988;30:556-560.

II. De Hamel FA, Welford B. Lung function in Maoris and Samoans working in New Zealand. N Z Med J 1983;96:560-562.

12. Ching B, Harsfall PAL. Lung volumes in Cantonese subjects:

Preliminary studies. Thorax 1977;32:352-355.

13. Da Costa JL. Pulmonary function studies in healthy Chinese adults in Singapure. Am Rev Respir Dis 1971;104:128-131.

14. Coultas D, Howard Ch, Skipper B, Samet J. Spirometric prediction equations for Hispanic children and adults in New Mexico. Am Rev Respir Dis 1988; |38: I386-1392.

15. Shafer BA, Samet JM, Coultas DB, Stidley CA. Prediction of lung function in Hispanics using local ethnic-specific and external non-ethnicspecific prediction equations. Am Rev Respir Dis 1993; 147: 1349-1353.

16. Crapo R, Morris A, Gardner R. Reference spirometric values using techniques and equipment that meet ATS recommendations. Am Rev Respir Dis 1981;123: 659-664.

17. Knudson R, Lebowitz M, Holberg C, Burrows B. Changes in the normal maximal expiratory flow-volume curve with growth and aging. Am Rev Respir Dis 1983;127:725-734.
18. Quanjer PH, Tammeling GJ, Cotes JE, Pedersen OF, Peslin R, Yemault JC. Standarized lung function testing. Lung volumes and forced ventilatory flows. Eur Respir J 1993; 6:5-40.

19. Pérez-Padilla JR, Regalado J,Vázquez-García JC. Reproducibilidad de espirometrías en trabajadores mexicanos y valores de referencia internacionales. Salud Publica Mex 200I;43: II3-I2I.

20. Hankinson JL, Odencrantz JR, Fedan KB. Spirometric reference values from a sample of the general US population. Am J Respir Crit Care Med 1999;159:179-187.

21. Ferris BG. Epidemiolgy standardization project. Am Rev Respir Dis 1978;118:1-118

22. Moran AO. Neumopatía asociada a la inhalación de humo de leña (NAIHL): descripción clínica, funcional, radiológica y patológica. Tesis de Maestría en Ciencias Médicas. México, D.F.: PUIS, UNAM, 1992.

23. Enright PL, Johnson LR, Connett JE, Voelker H, Buist S. Spirometry in the lung health study. Methods and quality control.Am Rev Respir Dis 1991;143:1215-1223.

24. Enright PL. Survilliance for lung disease. Quality assurance using computers and a team approach. Occup Med 1992;7:209-225.

25. Armitage P, Berry G. Statistical methods in medical research. 3a edición.Victoria,Australia: Blackwell Scientific Publications, 1994. 26. Xu X, Li B,Wang L. Gender difference in smoking effects on adult pulmonary function. Eur Respir J 1994;7:477-483.

27. Scanlon PD, Connet JE, Waller LA, Altose MD, Bailey WC, Buist SA et al. Smoking cessation and lung function in mild to moderate chronic obstructive pulmonary disease. Am J Respir Crit Care Med 2000;161:381-390.

28. Silverman EK, Weiss ST, Drazen JM, Chapman HA, Carey V, Campbell Ej et al. Gender related differences in severe, early onset chronic obstructive pulmonary disease. Am J Respir Crit Care Med 2000; |62:2152-2158.

29. Vollmer WM, Enright PL, Pedula KA, Speizer F, Kuller LH, Kiley J et al. Race and gender difference in the effects of smoking on lung function. Chest 2000; 1 17:764-772.

30. Raven PB, Taguchi S, Drinkwater BL. Anthropometric, spirometric and physiologic comparisons of migrant Japanese. Hum Biol 1977;46:483-489.

3I. Roca J, Sanchís J, Agustí-Vidal A, Segarra F, Navajas D, RodríguezRoisin $\mathrm{R}$ et al. Spirometric reference values from a Mediterranean population. Bull Eur Physiopathol Respir 1986;22:217-224.

33. Regalado J. Ecuaciones de predicción espirométrica en mujeres asintomáticas expuestas al humo de leña. Tesis en Neumología. México, D.F.: UNAM, 2000.

34. González-Camarena R, Carrasco-Sosa S, Gaitán M. Reliability of reference models for vital capacity in young mexican females. Rev Inv Clin 1993;45:57I-574. 